\title{
Eigenvectors and Reconstruction
}

\author{
Hongyu He * \\ Department of Mathematics \\ Louisiana State University, Baton Rouge, USA \\ hongyu@math.lsu.edu
}

Submitted: Jul 6, 2006; Accepted: Jun 14, 2007; Published: Jul 5, 2007

Mathematics Subject Classification: 05C88

\begin{abstract}
In this paper, we study the simple eigenvectors of two hypomorphic matrices using linear algebra. We also give new proofs of results of Godsil and McKay.
\end{abstract}

\section{Introduction}

We start by fixing some notations ( [HE1]). Let $A$ be a $n \times n$ real symmetric matrix. Let $A_{i}$ be the matrix obtaining by deleting the $i$-th row and $i$-th column of $A$. We say that two symmetric matrices $A$ and $B$ are hypomorphic if, for each $i, B_{i}$ can be obtained by simultaneously permuting the rows and columns of $A_{i}$. Let $\Sigma$ be the set of permutations. We write $B=\Sigma(A)$.

If $M$ is a symmetric real matrix, then the eigenvalues of $M$ are real. We write

$$
\operatorname{eigen}(M)=\left(\lambda_{1}(M) \geq \lambda_{2}(M) \geq \ldots \geq \lambda_{n}(M)\right) \text {. }
$$

If $\alpha$ is an eigenvalue of $M$, we denote the corresponding eigenspace by eigen $(M)$. Let $\mathbf{1}$ be the $n$-dimensional vector $(1,1, \ldots, 1)$. Put $\mathbf{J}=\mathbf{1}^{t} \mathbf{1}$. In [HE1], we proved the following theorem.

Theorem 1 ( [HE1]) Let $B$ and $A$ be two real $n \times n$ symmetric matrices. Let $\Sigma$ be a hypomorphism such that $B=\Sigma(A)$. Let $t$ be a real number. Then there exists an open interval $T$ such that for $t \in T$ we have

1. $\lambda_{n}(A+t \mathbf{J})=\lambda_{n}(B+t \mathbf{J})$;

2. eigen $_{\lambda_{n}}(A+t \mathbf{J})$ and eigen ${\overline{\lambda_{n}}}(B+t \mathbf{J})$ are both one dimensional;

*I would like to thank the referee for his valuable comments. 
3. eigen $_{\lambda_{n}}(A+t \mathbf{J})=$ eigen $_{\lambda_{n}}(B+t \mathbf{J})$.

As proved in [HE1], our result implies Tutte's theorem which says that eigen $(A+t J)=$ $\operatorname{eigen}(B+t J)$. So $\operatorname{det}(A+t J-\lambda I)=\operatorname{det}(B+t J-\lambda I)$.

In this paper, we shall study the eigenvectors of $A$ and $B$. Most of the results in this paper are not new. Our approach is new. We apply Theorem 1 to derive several wellknown results. We first prove that the squares of the entries of simple unit eigenvectors of $A$ can be reconstructed as functions of eigen $(A)$ and eigen $\left(A_{i}\right)$. This yields a proof of a Theorem of Godsil-McKay. We then study how the eigenvectors of $A$ change after a perturbation of rank 1 symmetric matrices. Combined with Theorem 1, we prove another result of Godsil-McKay which states that the simple eigenvectors that are perpendicular to $\mathbf{1}$ are reconstructible. We further show that the orthogonal projection of $\mathbf{1}$ onto higher dimensional eigenspaces is reconstructible.

Our investigation indicates that the following conjecture could be true.

Conjecture 1 Let $A$ be a real $n \times n$ symmetric matrix. Then there exists a subgroup $G(A) \subseteq O(n)$ such that a real symmetric matrix $B$ satisfies the properties that eigen $(B)=$ eigen $(A)$ and eigen $\left(B_{i}\right)=$ eigen $\left(A_{i}\right)$ for each $i$ if and only if $B=U A U^{t}$ for some $U \in G(A)$.

This conjecture is clearly true if $\operatorname{rank}(A)=1$. For $\operatorname{rank}(A)=1$, the group $G(A)$ can be chosen as $\mathbb{Z}_{2}^{n}$, all in the form of diagonal matrices. In some other cases, $G(A)$ can be a subgroup of the permutation group $S_{n}$.

\section{Reconstruction of Square Functions}

Theorem 2 Let $A$ be a $n \times n$ real symmetric matrix. Let $\left(\lambda_{1} \geq \lambda_{2} \geq \cdots \geq \lambda_{n}\right)$ be the eigenvalues of $A$. Suppose $\lambda_{i}$ is a simple eigenvalue of $A$. Let $\mathbf{p}_{i}=\left(p_{1, i}, p_{2, i}, \ldots, p_{n, i}\right)^{t}$ be a unit vector in eigen $\lambda_{\lambda_{i}}(A)$. Then for every $m, p_{m, i}^{2}$ can be expressed as a function of eigen $(A)$ and eigen $\left(A_{m}\right)$.

Proof: Let $\lambda_{i}$ be a simple eigenvalue of $A$. Let $\mathbf{p}_{i}=\left(p_{1, i}, p_{2, i}, \ldots, p_{n, i}\right)^{t}$ be a unit vector in eigen $_{\lambda_{i}}(A)$. There exists an orthogonal matrix $P$ such that $P=\left(\mathbf{p}_{1}, \mathbf{p}_{2}, \cdots, \mathbf{p}_{n}\right)$ and $A=P D P^{t}$ where

$$
D=\left(\begin{array}{cccc}
\lambda_{1} & 0 & \cdots & 0 \\
0 & \lambda_{2} & \cdots & 0 \\
\vdots & \vdots & \ddots & \vdots \\
0 & 0 & \cdots & \lambda_{n}
\end{array}\right)
$$

Then

$$
A-\lambda_{i} I=P D P^{t}-\lambda_{i} I=P\left(D-\lambda_{i} I\right) P^{t}=\sum_{j \neq i}\left(\lambda_{j}-\lambda_{i}\right) \mathbf{p}_{j} \mathbf{p}_{j}^{t}
$$


which equals

$$
\begin{gathered}
\left(\begin{array}{ccccc}
p_{1,1} & \cdots & \widehat{p_{1, i}} & \cdots & p_{1, n} \\
p_{2,1} & \cdots & \widehat{p_{2, i}} & \cdots & p_{2, n} \\
\vdots & \ddots & \vdots & \ddots & \vdots \\
p_{n, 1} & \cdots & \widehat{p_{n, i}} & \cdots & p_{n, n}
\end{array}\right)\left(\begin{array}{cccccc}
\lambda_{1}-\lambda_{i} & \cdots & 0 & \cdots & 0 \\
\vdots & \ddots & \vdots & \ddots & \vdots \\
0 & \cdots & \widehat{\lambda_{i}-\lambda_{i}} & \cdots & 0 \\
\vdots & \ddots & \vdots & \ddots & \vdots \\
0 & \cdots & 0 & \cdots & \lambda_{n}-\lambda_{i}
\end{array}\right) \\
\\
\left(\begin{array}{cccc}
p_{1,1} & p_{2,1} & \cdots & p_{n, 1} \\
\vdots & \vdots & \ddots & \vdots \\
\widehat{p_{1, i}} & \widehat{p_{2, i}} & \cdots & \widehat{p_{n, i}} \\
\vdots & \vdots & \ddots & \vdots \\
p_{1, n} & p_{2, n} & \cdots & p_{n, n}
\end{array}\right) .
\end{gathered}
$$

Deleting the $m$-th row and $m$-th column, we obtain

$$
\begin{aligned}
&\left(\begin{array}{ccccr}
p_{1,1} & \cdots & \widehat{p_{1, i}} & \cdots & p_{1, n} \\
\vdots & \ddots & \vdots & \ddots & \vdots \\
\widehat{p_{m, 1}} & \cdots & \widehat{p_{m, i}} & \cdots & \widehat{p_{m, n}} \\
\vdots & \ddots & \vdots & \ddots & \vdots \\
p_{n, 1} & \cdots & \widehat{p_{n, i}} & \cdots & p_{n, n}
\end{array}\right)\left(\begin{array}{cccccc}
\lambda_{1}-\lambda_{i} & \cdots & 0 & \cdots & 0 \\
\vdots & \ddots & \vdots & \ddots & \vdots \\
0 & & \cdots & \widehat{\lambda_{i}-\lambda_{i}} & \cdots & 0 \\
\vdots & \ddots & \vdots & \ddots & \vdots \\
0 & & \cdots & 0 & \cdots & \lambda_{n}-\lambda_{i}
\end{array}\right) \\
&\left(\begin{array}{ccccc}
p_{1,1} & \cdots & \widehat{p_{m, 1}} & \cdots & p_{n, 1} \\
\vdots & \ddots & \vdots & \ddots & \vdots \\
\widehat{p_{1, i}} & \cdots & \widehat{p_{m, i}} & \cdots & \widehat{p_{n, i}} \\
\vdots & \ddots & \vdots & \ddots & \vdots \\
p_{1, n} & \cdots & \widehat{p_{m, n}} & \cdots & p_{n, n}
\end{array}\right) .
\end{aligned}
$$

This is $A_{m}-\lambda_{i} I_{n-1}$. Notice that $P$ is orthogonal. Let $P_{m, i}$ be the matrix obtained by deleting the $m$-th row and $i$-th column. Then $\operatorname{det} P_{m, i}^{2}=p_{m, i}^{2}$ where $p_{m, i}$ is the $(m, i)$-th entry of $P$. Taking the determinant, we have

$$
\operatorname{det}\left(A_{m}-\lambda_{i} I_{n-1}\right)=p_{m, i}^{2} \prod_{j \neq i}\left(\lambda_{j}-\lambda_{i}\right) .
$$

It follows that

$$
p_{m, i}^{2}=\frac{\prod_{j=1}^{n-1}\left(\lambda_{j}\left(A_{m}\right)-\lambda_{i}\right)}{\prod_{j \neq i}\left(\lambda_{j}-\lambda_{i}\right)} .
$$

Q.E.D.

Corollary 1 Let $A$ and $B$ be two $n \times n$ real symmetric matrices. Suppose that eigen $(A)=$ eigen $(B)$ and eigen $\left(A_{i}\right)=$ eigen $\left(B_{i}\right)$. Let $\lambda_{i}$ be a simple eigenvalue of $A$ and $B$. Let 
$\mathbf{p}_{i}=\left(p_{1, i}, p_{2, i}, \ldots, p_{n, i}\right)^{t}$ be a unit vector in eigen $\bar{\lambda}_{i}(A)$ and $\mathbf{q}_{i}=\left(q_{1, i}, q_{2, i}, \ldots, q_{n, i}\right)^{t}$ be a unit vector in eigen ${\overline{\lambda_{i}}}(B)$. Then

$$
p_{j, i}^{2}=q_{j, i}^{2} \forall j \in[1, n]
$$

Corollary 2 (Godsil-McKay, see Theorem 3.2, [GM]) Let $A$ and $B$ be two $n \times n$ real symmetric matrices. Suppose that $A$ and $B$ are hypomorphic. Let $\lambda_{i}$ be a simple eigenvalue of $A$ and $B$. Let $\mathbf{p}_{i}=\left(p_{1, i}, p_{2, i}, \ldots, p_{n, i}\right)^{t}$ be a unit vector in eigen $\lambda_{\lambda_{i}}(A)$ and $\mathbf{q}_{i}=\left(q_{1, i}, q_{2, i}, \ldots, q_{n, i}\right)^{t}$ be a unit vector in eigen ${\lambda_{i}}(B)$. Then

$$
p_{j, i}^{2}=q_{j, i}^{2} \forall j \in[1, n] \text {. }
$$

\section{Eigenvalues and Eigenvectors under the perturba- tion of a rank one symmetric matrix}

Let $A$ be a $n \times n$ real symmetric matrix. Let $x$ be a $n$-dimensional row column vector. Let $M=x x^{t}$. Now consider $A+t M$. We have

$$
A+t M=P D P^{t}+t M=P\left(D+t P^{t} M P\right) P^{t}=P\left(D+t P^{t} x x^{t} P\right) P^{t} .
$$

Let $P^{t} x=q$. So $q_{i}=\left(\mathbf{p}_{i}, x\right)$ for each $i \in[1, n]$. Then

$$
A+t M=P\left(D+t q q^{t}\right) P^{t}
$$

Put $D(t)=D+t q q^{t}$.

Lemma $1 \operatorname{det}\left(D+t q q^{t}-\lambda I\right)=\operatorname{det}(A-\lambda I)\left(1+\sum_{i} \frac{t q_{i}^{2}}{\lambda_{i}-\lambda}\right)$.

Proof: $\operatorname{det}\left(D-\lambda I+t q q^{t}\right)$ can be written as a sum of products of $\lambda_{i}-\lambda$ and $q_{i} q_{j}$. For each $S$ a subset of $[1, n]$, combine the terms containing only $\prod_{i \in S}\left(\lambda_{i}-\lambda\right)$. Since the rank of $q q^{t}$ is one, only for $|S|=n, n-1$, the coefficients may be nonzero. We obtain

$$
\operatorname{det}\left(D+t q q^{t}-\lambda I\right)=\prod_{i=1}^{n}\left(\lambda_{i}-\lambda\right)+\sum_{i=1}^{n} t q_{i}^{2} \prod_{j \neq i}\left(\lambda_{i}-\lambda\right) .
$$

The Lemma follows.

Put $P_{t}(\lambda)=1+\sum_{i} \frac{t q_{i}^{2}}{\lambda_{i}-\lambda}$.

Lemma 2 Fix $t<0$. Suppose that $\lambda_{1}, \lambda_{2}, \ldots, \lambda_{n}$ are distinct and $q_{i} \neq 0$ for every $i$. Then $P_{t}(\lambda)$ has exactly $n$ roots $\left(\mu_{1}, \mu_{2}, \cdots, \mu_{n}\right)$ satisfying an interlacing relation:

$$
\lambda_{1}>\mu_{1}>\lambda_{2}>\mu_{2}>\cdots>\mu_{n-1}>\lambda_{n}>\mu_{n} .
$$


Proof: Clearly, $\frac{d P_{t}(\lambda)}{d \lambda}=\sum_{i} \frac{t q_{i}^{2}}{\left(\lambda_{i}-\lambda\right)^{2}}<0$. So $P_{t}(\lambda)$ is always decreasing. On the interval $\left(-\infty, \lambda_{n}\right), \lim _{\lambda \rightarrow-\infty} P_{t}(\lambda)=1$ and $\lim _{\lambda \rightarrow \lambda_{n}^{-}} P_{t}(\lambda)=-\infty$. So $P_{t}(\lambda)$ has a unique root $\mu_{n} \in\left(-\infty, \lambda_{n}\right)$. Similar statement holds for each $\left(\lambda_{i-1}, \lambda_{i}\right)$. On $\left(\lambda_{1}, \infty\right), \lim _{\lambda \rightarrow \infty} P_{t}(\lambda)=1$ and $\lim _{\lambda \rightarrow \lambda_{1}^{+}} P_{t}(\lambda)=\infty$. So $P_{t}(\lambda)$ does not have any roots in $\left(\lambda_{1}, \infty\right)$. Q.E.D.

Theorem 3 Fix $t<0$ and $x \in \mathbb{R}^{n}$. Let $M=x x^{t}$. Let $l$ be the number of distinct eigenvalues satisfying $\left(x\right.$, eigen $\left._{\lambda}(A)\right) \neq 0$. Choose an orthonormal basis of each eigenspace of $A$ so that one of the eigenvectors is a multiple of the orthogonal projection of $x$ onto the eigenspace if this projection is nonzero. Denote this basis by $\left\{\mathbf{p}_{i}\right\}$ and let $P=\left(\mathbf{p}_{1}, \mathbf{p}_{2}, \ldots, p_{n}\right)$. Let

$$
S=\left\{i_{1}>i_{2}>\cdots>i_{l}\right\}
$$

such that $\left(x, \mathbf{p}_{i}\right) \neq 0$ for every $i \in S$ and $\left(x, \mathbf{p}_{i}\right)=0$ for every $i \notin S$. Then there exists $\left(\mu_{1}, \ldots, \mu_{l}\right)$ such that

$$
\lambda_{i_{1}}>\mu_{1}>\lambda_{i_{2}}>\mu_{2}>\cdots>\lambda_{i_{l}}>\mu_{l}
$$

and

$$
\operatorname{eigen}(A+t M)=\left\{\lambda_{i}(A) \mid i \notin S\right\} \cup\left\{\mu_{1}, \mu_{2} \ldots, \mu_{l}\right\} .
$$

Furthermore, eigen $\mu_{j}(A+t M)$ contains

$$
\sum_{i \in S} \mathbf{p}_{i} \frac{q_{i}}{\lambda_{i}-\mu_{j}}
$$

Here the index set $\left\{i_{1}, i_{2}, \cdots, i_{l}\right\}$ may not be unique. I shall also point out a similar statement holds for $t>0$ with

$$
\mu_{1}>\lambda_{i_{1}}>\mu_{2}>\lambda_{i_{2}}>\cdots>\mu_{l}>\lambda_{i_{l}} .
$$

Proof: Recall that $q_{i}=\left(\mathbf{p}_{i}, x\right)$. Since $\left(x\right.$, eigen $\left._{\lambda_{i_{j}}}(A)\right) \neq 0, q_{i_{j}} \neq 0$. For $i \notin S, q_{i}=0$. Notice

$$
P_{t}(\lambda)=1+\sum_{j=1}^{l} \frac{t q_{i_{j}}^{2}}{\lambda_{i_{j}}-\lambda} .
$$

Applying Lemma 2 to $S$, we obtain the roots of $P_{t}(\lambda),\left\{\mu_{1}, \mu_{2}, \ldots, \mu_{l}\right\}$, satisfying

$$
\lambda_{i_{1}}>\mu_{1}>\lambda_{i_{2}}>\mu_{2}>\cdots>\lambda_{i_{l}}>\mu_{l} .
$$

It follows that the roots of $\operatorname{det}(A+t M-\lambda I)=P_{t}(\lambda) \prod_{i=1}^{n}\left(\lambda_{i}-\lambda\right)$ can be obtained from eigen $(A)$ be changing $\left\{\lambda_{i_{1}}>\lambda_{i_{2}}>\cdots>\lambda_{i_{l}}\right\}$ to $\left\{\mu_{1}, \mu_{2} \ldots, \mu_{l}\right\}$. Therefore,

$$
\operatorname{eigen}(A+t M)=\left\{\lambda_{i}(A) \mid i \notin S\right\} \cup\left\{\mu_{1}, \mu_{2} \ldots, \mu_{l}\right\} .
$$


Fix a $\mu_{j}$. Let $\left\{\mathbf{e}_{i}\right\}$ be the standard basis for $\mathbb{R}^{n}$. Notice that

$$
\begin{aligned}
& (A+t M) \sum_{i \in S} \frac{q_{i}}{\lambda_{i}-\mu_{j}} \mathbf{p}_{i} \\
= & P\left(D+t q q^{t}\right) P^{t} \sum_{i \in S} \frac{q_{i}}{\lambda_{i}-\mu_{j}} \mathbf{p}_{i} \\
= & P\left(D+t q q^{t}\right) \sum_{i \in S} \frac{q_{i}}{\lambda_{i}-\mu_{j}} \mathbf{e}_{i} \\
= & P\left(\sum_{i \in S} \frac{\lambda_{i} q_{i}}{\lambda_{i}-\mu_{j}} \mathbf{e}_{i}+t\left(\begin{array}{c}
q_{1} \\
\vdots \\
q_{n}
\end{array}\right) \sum_{i \in S} \frac{q_{i}^{2}}{\lambda_{i}-\mu_{j}}\right) \\
= & P\left(\sum_{i \in S} \frac{\lambda_{i} q_{i}}{\lambda_{i}-\mu_{j}} \mathbf{e}_{i}-\sum_{i \in S} q_{i} \mathbf{e}_{i}\right) \\
= & P \sum_{i \in S} \frac{\mu_{j} q_{i}}{\lambda_{i}-\mu_{j}} \mathbf{e}_{i} \\
= & \mu_{j} \sum_{i \in S} \frac{q_{i}}{\lambda_{i}-\mu_{j}} \mathbf{p}_{i}
\end{aligned}
$$

Notice that here we use the fact that $P_{t}\left(\mu_{j}\right)=\sum_{i \in S} \frac{t q_{i}^{2}}{\lambda_{i}-\mu_{j}}+1=0$. We have obtained that $(A+t M) \sum_{\lambda_{i} \in S} \frac{q_{i}}{\lambda_{i}-\mu_{j}} \mathbf{p}_{i}=\mu_{j} \sum_{i \in S} \frac{q_{i}}{\lambda_{i}-\mu_{j}} \mathbf{p}_{i}$. Therefore,

$$
\sum_{i \in S} \frac{q_{i}}{\lambda_{i}-\mu_{j}} \mathbf{p}_{i} \in \operatorname{eigen}_{\mu_{j}}(A+t M) \text {. }
$$

Q.E.D.

\section{Reconstruction of Simple Eigenvectors not perpen- dicular to 1}

Now let $M=\mathbf{J}=\mathbf{1 1}^{t}$. Theorem 3 applies to $A+t \mathbf{J}$ and $B+t \mathbf{J}$.

Theorem 4 (Godsil-McKay, [GM]) Let $B$ and $A$ be two real $n \times n$ symmetric matrices. Let $\Sigma$ be a hypomorphism such that $B=\Sigma(A)$. Let $S \subseteq[1, n], A=P D P^{t}$ and $B=U D U^{t}$ be as in Theorem 3. For $i \in S$, we have $\mathbf{p}_{i}=\mathbf{u}_{i}$ or $\mathbf{p}_{i}=-\mathbf{u}_{i}$. In particular, if $\lambda_{i}$ is a simple eigenvalue of $A$ and $\left(\right.$ eigen $\left._{\lambda_{i}}(A), \mathbf{1}\right) \neq 0$, then eigen $\lambda_{\lambda_{i}}(A)=$ eigen $_{\lambda_{i}}(B)$.

Proof: • By Tutte's theorem, eigen $(A)=\operatorname{eigen}(B)$. Let $A=P D P^{t}$ and $B=U D U^{t}$. Since $\operatorname{det}(A+t \mathbf{J}-\lambda I)=\operatorname{det}(B+t \mathbf{J}-\lambda I)$, by Lemma 1 ,

$$
\operatorname{det}(A-\lambda I)\left(1+\sum_{i} \frac{t\left(\mathbf{1}, \mathbf{p}_{i}\right)^{2}}{\lambda_{i}-\lambda}\right)=\operatorname{det}(B-\lambda I)\left(1+\sum_{i} \frac{t\left(\mathbf{1}, \mathbf{u}_{i}\right)^{2}}{\lambda_{i}-\lambda}\right) .
$$


It follows that for every $\lambda_{i}, \sum_{\lambda_{j}=\lambda_{i}}\left(\mathbf{1}, \mathbf{p}_{j}\right)^{2}=\sum_{\lambda_{j}=\lambda_{i}}\left(\mathbf{1}, \mathbf{u}_{j}\right)^{2}$. Consequently, the $l$ for $A$ is the same as the $l$ for $B$. Let $S$ be as in Theorem 3 for both $A$ and $B$. Without loss of generality, suppose that $A=P D P^{t}$ and $B=U D U^{t}$ as in Theorem 3. In particular, for every $i \in[1, n]$, we have

$$
\left(\mathbf{p}_{i}, \mathbf{1}\right)^{2}=\left(\mathbf{u}_{i}, \mathbf{1}\right)^{2} .
$$

- Let $T$ be as in the proof of Theorem 1 in [HE1] for $A$ and $B$. Without loss of generality, suppose $T=\left(t_{1}, t_{2}\right) \subseteq \mathbb{R}^{-}$. Let $t \in T$ and let $\mu_{l}(t)$ be the $\mu_{l}$ in Theorem 3 for $A$ and $B$. Notice that the lowest eigenvectors of $A+t \mathbf{J}$ and $B+t \mathbf{J}$ are in $\mathbb{R}^{+n}$ (see Lemma 1, Theorem 7 and Proof of Theorem 2 in [HE1]). So they are not perpendicular to 1. By Theorem 3, $\mu_{l}(t)=\lambda_{n}(A+t \mathbf{J})=\lambda_{n}(B+t \mathbf{J})$. By Theorem 1,

$$
\text { eigen }_{\mu_{1}(t)}(A+t \mathbf{J})=\text { eigen }_{\mu_{l}(t)}(B+t \mathbf{J}) \cong \mathbb{R} .
$$

So $\sum_{i \in S} \mathbf{p}_{i} \frac{\left(\mathbf{p}_{i}, \mathbf{1}\right)}{\lambda_{i}-\mu_{l}(t)}$ is parallel to $\sum_{i \in S} \mathbf{u}_{i} \frac{\left(\mathbf{u}_{i}, \mathbf{1}\right)}{\lambda_{i}-\mu_{l}(t)}$. Since $\left\{\mathbf{p}_{i}\right\}$ and $\left\{\mathbf{u}_{i}\right\}$ are orthonormal, by Equation 2,

$$
\left\|\sum_{i \in S} \mathbf{p}_{i} \frac{\left(\mathbf{p}_{i}, \mathbf{1}\right)}{\lambda_{i}-\mu_{l}(t)}\right\|^{2}=\left\|\sum_{i \in S} \mathbf{u}_{i} \frac{\left(\mathbf{u}_{i}, \mathbf{1}\right)}{\lambda_{i}-\mu_{l}(t)}\right\|^{2} .
$$

It follows that for every $t \in T$,

$$
\sum_{i \in S} \mathbf{p}_{i} \frac{\left(\mathbf{p}_{i}, \mathbf{1}\right)}{\lambda_{i}-\mu_{l}(t)}= \pm \sum_{i \in S} \mathbf{u}_{i} \frac{\left(\mathbf{u}_{i}, \mathbf{1}\right)}{\lambda_{i}-\mu_{l}(t)}
$$

- Recall that $-\frac{1}{t}=\sum_{i} \frac{q_{i}^{2}}{\lambda_{i}-\mu_{l}(t)}$. Notice that the function $\rho \rightarrow \sum_{i} \frac{q_{i}^{2}}{\lambda_{i}-\rho}$ is a continuous and one-to-one mapping from $\left(-\infty, \lambda_{n}\right)$ onto $(0, \infty)$. There exists a nonempty interval $T_{0} \subseteq\left(-\infty, \lambda_{n}\right)$ such that if $\rho \in T_{0}$, then $\sum_{i} \frac{q_{i}^{2}}{\lambda_{i}-\rho} \in\left(-\frac{1}{t_{1}},-\frac{1}{t_{2}}\right)$. So every $\rho \in T_{0}$ is a $\mu_{l}(t)$ for some $t \in\left(t_{1}, t_{2}\right)$. It follow that for every $\rho \in T_{0}$,

$$
\sum_{i \in S} \mathbf{p}_{i} \frac{\left(\mathbf{p}_{i}, \mathbf{1}\right)}{\lambda_{i}-\rho}= \pm \sum_{i \in S} \mathbf{u}_{i} \frac{\left(\mathbf{u}_{i}, \mathbf{1}\right)}{\lambda_{i}-\rho}
$$

Notice that both vectors are nonzero and depend continuously on $\rho$. Either,

$$
\sum_{i \in S} \mathbf{p}_{i} \frac{\left(\mathbf{p}_{i}, \mathbf{1}\right)}{\lambda_{i}-\rho}=\sum_{i \in S} \mathbf{u}_{i} \frac{\left(\mathbf{u}_{i}, \mathbf{1}\right)}{\lambda_{i}-\rho} \quad \forall\left(\rho \in T_{0}\right) ;
$$

or,

$$
\sum_{i \in S} \mathbf{p}_{i} \frac{\left(\mathbf{p}_{i}, \mathbf{1}\right)}{\lambda_{i}-\rho}=-\sum_{i \in S} \mathbf{u}_{i} \frac{\left(\mathbf{u}_{i}, \mathbf{1}\right)}{\lambda_{i}-\rho} \quad \forall\left(\rho \in T_{0}\right) ;
$$

- Notice that the functions $\left.\left\{\rho \rightarrow \frac{1}{\lambda_{i_{j}}-\rho}\right\}\right|_{i_{j} \in S}$ are linearly independent. For every $i \in S$, we have

$$
\mathbf{p}_{i}\left(\mathbf{p}_{i}, \mathbf{1}\right)= \pm \mathbf{u}_{i}\left(\mathbf{u}_{i}, \mathbf{1}\right)
$$

Because $\mathbf{p}_{i}$ and $\mathbf{u}_{i}$ are both unit vectors, $\mathbf{p}_{i}= \pm \mathbf{u}_{i}$. In particular, for every simple $\lambda_{i}$ with $\left(\mathbf{p}_{i}, \mathbf{1}\right) \neq 0$ we have eigen ${\lambda_{i}}(A)=$ eigen $_{\lambda_{i}}(B)$. Q.E.D. 
Corollary 3 Let $B$ and $A$ be two real $n \times n$ symmetric matrices. Suppose that $B=\Sigma(A)$ for a hypomorphism $\Sigma$. Let $\lambda_{i}$ be an eigenvalue of $A$ such that $\left(\right.$ eigen $\left._{\lambda_{i}}(A), \mathbf{1}\right) \neq 0$. Then the orthogonal projection of $\mathbf{1}$ onto eigen $\lambda_{\lambda_{i}}(A)$ equals the orthogonal projection of $\mathbf{1}$ onto eigen $_{\lambda_{i}}(B)$.

Proof: Notice that the projections are $\mathbf{p}_{i}\left(\mathbf{p}_{i}, \mathbf{1}\right)$ and $\mathbf{u}_{i}\left(\mathbf{u}_{i}, \mathbf{1}\right)$. Whether $\mathbf{p}_{i}=\mathbf{u}_{i}$ or $\mathbf{p}_{i}=$ $-\mathbf{u}_{i}$, we always have

$$
\mathbf{p}_{i}\left(\mathbf{p}_{i}, \mathbf{1}\right)=\mathbf{u}_{i}\left(\mathbf{u}_{i}, \mathbf{1}\right)
$$

Q.E.D.

Conjecture 2 Let $A$ and $B$ be two hypomorphic matrices. Let $\lambda_{i}$ be a simple eigenvalue of $A$. Then there exists a permutation matrix $\tau$ such that $\tau$ eigen $n_{\lambda_{i}}(A)=$ eigen $_{\lambda_{i}}(B)$.

This conjecture is apparently true if $\operatorname{eigen}_{\lambda_{i}}(A)$ is not perpendicular to $\mathbf{1}$.

\section{References}

[Tutte] W. T. Tutte, "All the King's Horses (A Guide to Reconstruction)", Graph Theory and Related Topics, Academic Press, 1979, (15-33).

[GM] C. D. Godsil and B. D. McKay, "Spectral Conditions for the Reconstructiblity of a graph", J. Combin. Theory Ser. B 30. 1981, No. 3, (285-289).

[HE1] H. He, "Reconstruction and Higher Dimensional Geometry", Journal of Combinatorial Theory, Series B 97, No 3 (421-429).

[Ko] W. Kocay, "Some New Methods in Reconstruction Theory", Combinatorial mathematics, IX (Brisbane, 1981), LNM 952, (89-114). 